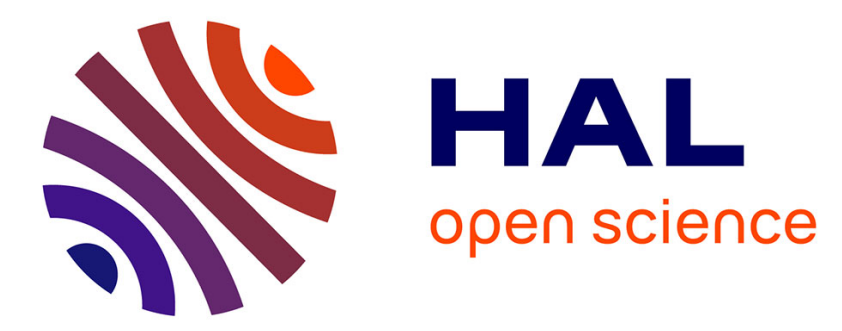

\title{
Multistatic and Multiple Frequency Imaging Resolution Analysis-Application to GPS-Based Multistatic Radar
}

Franck Daout, Françoise Schmitt, Guillaume Ginolhac, Philippe Fargette

\section{To cite this version:}

Franck Daout, Françoise Schmitt, Guillaume Ginolhac, Philippe Fargette. Multistatic and Multiple Frequency Imaging Resolution Analysis-Application to GPS-Based Multistatic Radar. IEEE Transactions on Aerospace and Electronic Systems, 2012, 48 (4), pp.3042 - 3057. 10.1109/TAES.2012.6324676 . hal-00828343

\section{HAL Id: hal-00828343 \\ https://hal.science/hal-00828343}

Submitted on 5 Jun 2013

HAL is a multi-disciplinary open access archive for the deposit and dissemination of scientific research documents, whether they are published or not. The documents may come from teaching and research institutions in France or abroad, or from public or private research centers.
L'archive ouverte pluridisciplinaire HAL, est destinée au dépôt et à la diffusion de documents scientifiques de niveau recherche, publiés ou non, émanant des établissements d'enseignement et de recherche français ou étrangers, des laboratoires publics ou privés. 


\title{
Multistatic and Multiple Frequency Imaging Resolution
}

\section{Analysis - Application to GPS-based Multistatic Radar}

\author{
F. Daout*, F. Schmitt*, G.Ginolhac* and P. Fargette**
}

October 19, 2011

*SATIE, ENS Cachan, CNRS, Universud, 61 av du Président Wilson 94235 CACHAN Cedex, France.

**DEMR, ONERA, Palaiseau, France.

\begin{abstract}
This paper focuses on the computation of the Generalized Ambiguity Function (GAF) of a Multiple Antennas Multiple Frequencies Radar system (MAMF). This study provides some insights into the definition of resolution parameters of a MAMF Radar system. It turns out that the range and azimuth resolutions are not the most suitable criteria to specify the MAMF Radar resolution. Therefore a new set of resolution parameters is introduced like the resolution ellipse which expresses the resolution anywhere in the image plane or $\vec{\delta}_{\text {max }}$, $\left(\vec{\delta}_{\text {min }}\right)$ which expresses the highest (lowest) bound of the spatial Radar resolution. To point out the pertinence of our study, we illustrate it with a MAMF Radar system built around GPS satellites. The effect of the Radar system geometry on resolution is investigated. For several scenarios, the General Ambiguity Function (GAF) and its numerical form, the Point Spread Function (PSF), are computed and their results are compared.
\end{abstract}

Keywords: Ambiguity Function, Point Spread Function, Resolution, Bistatic SAR, Multistatic SAR, Passive SAR.

\section{Introduction}

Monostatic Radar systems where the transmitting and the receiving antennas are located on the same platform, have been the subject of a great deal of research and development over the past 
few decades. Their spatial performances are well known: the range resolution is determined by the frequency bandwidth and the azimuth resolution is defined from the integration time and the carrier frequency [1]. Recently, a growing interest in bistatic and multistatic Radar system has appeared (MAMF Radar system) [2, 3, 4, 5, 6, 7, 8]. A bistatic Radar is defined as a Radar using antennas at different locations for transmission and reception. A Multistatic Radar can be considered as a set of $M_{s}$ bistatic Radar. The MAMF geometry fits well with the use of non-cooperative transmitters such as TV or FM broadcasts [9, 10, 11, 12] or GNSS signals and allows for example the discreet survey of a specific zone.

Several papers treat of bistatic and/or multistatic radar performance subject. For example Tsao and al. [13] compute the ambiguity function for a bistatic radar. They show that the bistatic geometry plays a role in the shape of the ambiguity function. Two examples are provided to compare the monostatic ambiguity function with the bistatic one. Plots in delay-Doppler plane for the monostatic ambiguity function and in range-velocity plane for the bistatic ambiguity function show the effect of the bistatic geometry on the radar performances. Bradaric and al. [14] define an ambiguity function for a single transmitter, multiple receivers system. The ambiguity response is determined as a function of the range from the transmitter and of the relative speed in a fixed direction. The authors address the rules to select the weights for fusing multiple receiver signals in order to reach specified performances. Sensor placement in bistatic and multistatic radar systems and how it relates to the system resolution has been studied in [15], [16] and [17] by calculating the Cramer-Rao lower bounds (CRLBs) for bistatic radar channel.

In this paper, we evaluate the displacement effect of the radar system on its ability to separate two very close motionless targets. We measure this effect according to the transmitter and receiver trajectories and to the signal characteristics. We start with the Generalized Ambiguity Function (GAF) in the bistatic context. Here, the ambiguity function is not analyzed with the usual delay and Doppler variables and the performances are not described in term of range-velocity parameters; we chose to work in the $(x, y)$ plane in order to give tools to a radar engineer to evaluate the spatial performances from a given multistatic Radar. Then the ellipse resolution is plotted in the $(x, y)$ plane. This ellipse is defined as the contour line at $1 / \sqrt{2}$ of the map of the normalized ambiguity function. In this way, we expand the work of [13] and show the same result : the ambiguity function needs to be considered with respect to the bistatic geometry ; plot in the Delay-Doppler plane is not efficient. In the $(x, y)$ plane, we derive formulas to evaluate the maximum and minimum spatial resolutions $\left(\vec{\delta}_{\text {max }}, \vec{\delta}_{\text {min }}\right)$. They express the bounds on the spatial resolution of a bistatic Radar system.

From the bistatic case we expand the definition of the resolution parameters to the multistatic 
case. The incoherent summation of the bistatic ambiguity functions leads to equation (21) which is equivalent to the multistatic ambiguity function described in [14]. An additional contribution of our work comparing to [14] is the second ways to fuse the bistatic data : the coherent summation of the ambiguity functions. Note that here again we define all the resolution parameters in the $(x, y)$ plane.

An outline of this paper is as follows. In section II, we describe a MAMF Radar system. Section III studies the performances of a bistatic Radar system. Under several assumptions, we analytically characterize bistatic Radar resolution by evaluating the bistatic Generalized Ambiguity Function (GAF) [18]. Section IV generalizes the bistatic study to the multistatic case. We point out the effect of several transmitters on the resolution parameters. In this part we introduce new pertinent parameters to characterize the spatial resolution of a MAMF Radar systems. We summarize our conclusions in section V. In section VI, we evaluate the pertinence of our method : the system impulse response (Point Spread Function) of a MAMF Radar passive system based on GPS signal is numerically computed via the PFA algorithm [19]. GAF and PSF results are then compared. Finally we summarize our conclusions in section VII.

\section{System overview}

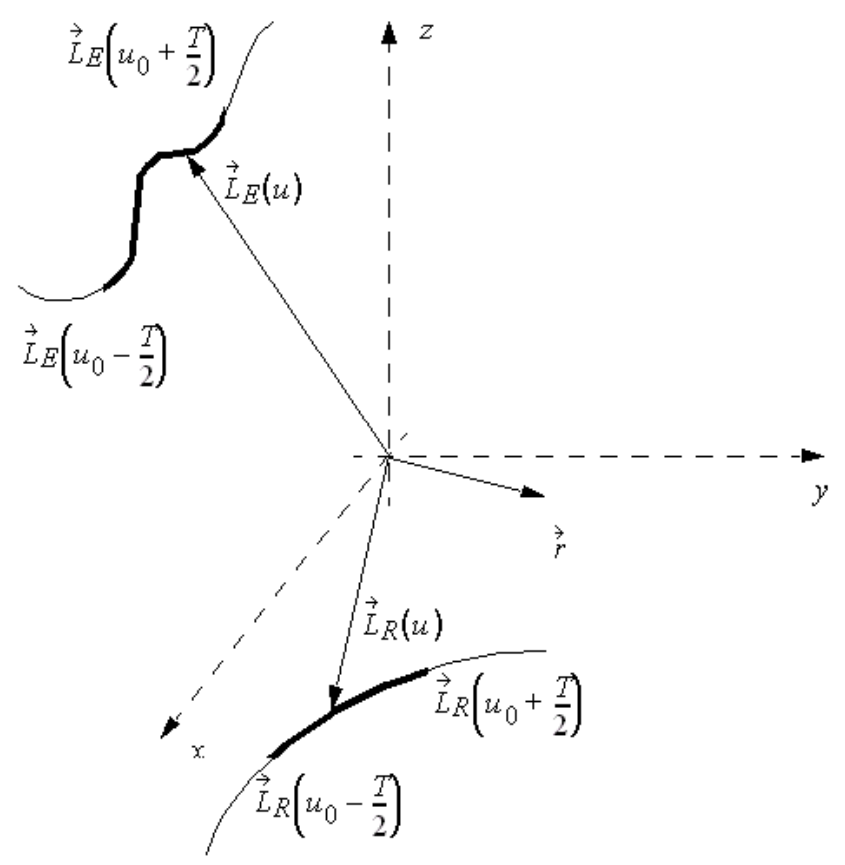

Figure 1: Bistatic geometry. The $(x, y)$ plane is the ground 
The aim of this study is to apply bistatic imaging methods to a multistatic context. Figure 1 shows a bistatic configuration with a motionless target, a transmitter located at $\vec{L}_{E}$ and a receiver located at $\vec{L}_{R}$. The displacement of the transmitter and (or) the receiver during the integration time $T$ forms the synthetic aperture. The localization of the transmitter and of the receiver at time $u$ along the aperture is given by $\vec{L}_{E}(u)$ and by $\vec{L}_{R}(u)$. The variable $u$ is called long time, in contrast with $t$ the wave propagation time, called the fast time. The multistatic configuration is defined as a combination of several bistatic configurations.

The center of the illuminated area is arbitrarily set at the origin. The following vectors are defined:

- $\vec{L}_{E}(u)=\left[\begin{array}{lll}L_{E}(u) \cos \theta_{e}(u) \cos \varphi_{e}(u) & L_{E}(u) \cos \theta_{e}(u) \sin \varphi_{e}(u) \quad L_{E}(u) \sin \theta_{e}(u)\end{array}\right]^{T}$ is the transmitter location,

- $\vec{L}_{R}(u)=\left[\begin{array}{lll}L_{R}(u) \cos \theta_{r}(u) \cos \varphi_{r}(u) & L_{R}(u) \cos \theta_{r}(u) \sin \varphi_{r}(u) \quad L_{R}(u) \sin \theta_{r}(u)\end{array}\right]^{T}$ is the receiver location,

- $\hat{L}_{E}(u)=\vec{L}_{E}(u) /\left\|\vec{L}_{E}(u)\right\|$ is the unit vector in the transmitter direction,

- $\hat{L}_{R}(u)=\vec{L}_{R}(u) /\left\|\vec{L}_{R}(u)\right\|$ is the unit vector in the direction of receiver,

- $\vec{\beta}(u)=\hat{L}_{E}(u)+\hat{L}_{R}(u)$ is the bisector of the bistatic angle $\beta=\left(\widehat{\hat{L}_{E}, \hat{L}_{R}}\right) \in[-\pi, \pi]$ (figure 2). Its magnitude is given by:

$$
\|\vec{\beta}(u)\|=2 \cos \left(\frac{\beta(u)}{2}\right)
$$

- $\hat{\beta}(u)=\left[\begin{array}{lll}\cos \gamma(u) \cos \alpha(u) & \sin \gamma(u) \cos \alpha(u) & \sin \alpha(u)\end{array}\right]^{T}=\frac{\vec{\beta}(u)}{\|\vec{\beta}(u)\|}$, is the unit vector in the direction of vector $\vec{\beta}$.

- $\hat{\beta}_{g}(u)=\left[\begin{array}{lll}\cos \gamma(u) & \sin \gamma(u) & 0\end{array}\right]^{T}$, and $\hat{\beta}_{g} \cos \alpha$ is the projection of $\hat{\beta}$ on the $(x, y)$ plane. 


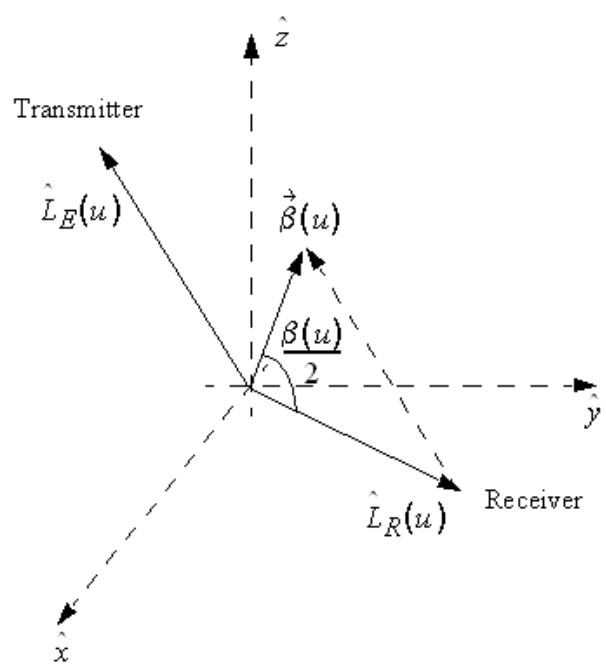

Figure 2: Direction and magnitude of $\vec{\beta}$

For monostatic systems, the transmitter and receiver are located at the same position. Therefore $\hat{L}_{E}=\hat{L}_{R}$ and the bistatic angle $\beta$ is equal to 0 . For a MAMF Radar system, the bistatic angle is non-zero and varies. This effect is studied in the next paragraph.

In the following, $k=2 \pi f / c$ denotes the wave-number, $\lambda=c / f$ is the wavelength, $f$ is the frequency and $c$ the speed of light.

\section{Bistatic Generalized Ambiguity Function}

For a monostatic Radar system, spatial performance is given by two parameters: range and azimuth resolutions $\left(\delta_{r}, \delta_{a}\right)$. Range resolution is related to the transmitted pulse width (or its frequency bandwidth $B$ ) [1]. Azimuth resolution is determined by the integration time $T$ and by the carrier frequency $f_{0}$. In the bistatic case, we define the vector $\vec{\delta}$ which describes the contour line at $1 / \sqrt{2}$ of the map of the normalized ambiguity function. The vector $\vec{\delta}$ describes an ellipse called the resolution ellipse. This ellipse is fully defined by its semi major axis $\vec{\delta}_{\max } / 2=\max (\vec{\delta})$ and by $\vec{\delta}_{\min } / 2=\min (\vec{\delta})$ its semi minor axis.

For a monostatic Radar system, the relationships between $\left(\delta_{r}, \delta_{a}\right)$ and $\left(\left\|\vec{\delta}_{\text {max }}\right\|,\left\|\vec{\delta}_{\text {min }}\right\|\right)$, are obvious. In fact $\left(\delta_{r}, \delta_{a}\right)=\left(\left\|\vec{\delta}_{\text {max }}\right\|,\left\|\vec{\delta}_{\text {min }}\right\|\right)$ (or $\left.\left(\left\|\vec{\delta}_{\text {min }}\right\|,\left\|\vec{\delta}_{\text {max }}\right\|\right)\right)$. In the case of a bistatic Radar system, the relationships between the parameters of interest $\left(\left\|\vec{\delta}_{\max }\right\|,\left\|\vec{\delta}_{\min }\right\|\right)$ and $\left(\delta_{r}, \delta_{a}\right)$ are not obvious because of the bistatic geometry. In this section, we evaluate the spatial performance of a bistatic system by computing $\left(\vec{\delta}_{\max }, \vec{\delta}_{\min }\right)$. 


\subsection{Generalized Ambiguity Function (GAF)}

Usually, for a monostatic Radar system, the Radar ambiguity function is defined as [20]:

$$
\left|\chi\left(\tau_{d}, f_{d}\right)\right|=\left|\int p(t) p^{*}\left(t+\tau_{d}\right) \exp \left(j 2 \pi f_{d} t\right) d t\right|
$$

where $p(t)$ is the complex signal envelope, $\tau_{d}$ the differential delay and $f_{d}$ the Doppler frequency. The notion of Radar ambiguity function has been extended to the bistatic Radar by Tao Zeng and al. [18]. Thus they define a Generalized Ambiguity Function (GAF) as:

$$
\chi(\overrightarrow{O A}, \overrightarrow{O B})=K \int s_{A}(t, u) s_{B}^{*}(t, u) d t d u
$$

where $s_{A}(t, u)$ and $s_{B}(t, u)$ are the base-band signals returned by a point target with unit Radar cross section and located at the ground points A and B. $K$ is used as normalization parameter such as $|\chi(\overrightarrow{O A}, \overrightarrow{O A})|=1$.

The distance between points $\mathrm{A}$ and $\mathrm{B}$ is small comparing to the distances transmitter-target and target-receiver. With an hypothesis of narrow band for the transmitted signal, equation (3) becomes (demonstration given in appendix 9.1):

$$
\chi(\vec{r})=K_{1} g\left(\tau_{d}(\vec{r})\right) M\left(f_{d}(\vec{r})\right)
$$

with $K_{1}$ a normalization constant and $\vec{r}=\overrightarrow{O A}-\overrightarrow{O B}=\overrightarrow{B A}$.

Equation (4) corresponds to the matched filter output of the receiver when a delay $\tau_{d}$ and a Doppler frequency $f_{d}$ occur. Consequently, it gives the Radar ability to separate two close targets separated by $\vec{r}$. Therefore the spatial resolution must be investigated by studying (4).

The differential delay $\tau_{d}$ is related to the geometry by:

$$
\tau_{d}=\frac{\vec{\beta}\left(u_{0}\right) \cdot \vec{r}}{c}=\frac{2 \cos \frac{\beta\left(u_{0}\right)}{2}\left(\hat{\beta}\left(u_{0}\right) \cdot \vec{r}\right)}{c}
$$

$u_{0}$ is the value of the long time $u$ evaluated at the midpoint of the integration time $T$ (i.e. $u \in$ $\left.\left[u_{0}-T / 2, u_{0}+T / 2\right]\right)$. Function $g(t)$ is related to the inverse Fourier transform of $\left|P\left(f-f_{0}\right)\right|^{2}$ the demodulated signal power spectrum. We assume that the transmitted pulses $P(f)$ have uniform power $P_{0}$ over the frequency range: $f \in\left[f_{0}-B / 2, f_{0}+B / 2\right]$. With this assumption, $g\left(\tau_{d}\right)$ is given by: 


$$
g\left(\tau_{d}\right)=P_{0} B \frac{\sin \left(\pi B \tau_{d}\right)}{\pi B \tau_{d}}
$$

Parameter $f_{d}$ is the differential Doppler frequency:

$$
f_{d}=f_{0} \frac{d \tau_{d}}{d u}=\frac{1}{\lambda_{0}} \frac{d \vec{\beta}\left(u_{0}\right)}{d u} \cdot \vec{r}
$$

with $\lambda_{0}=c / f_{0}$. Function $M\left(f_{d}\right)$ is the inverse Fourier transform of $m(u)$, constant during the integration time $T$ (The antennas are supposed to be isotropic):

$$
M\left(f_{d}\right)=T \frac{\sin \pi T f_{d}}{\pi T f_{d}}
$$

In the next paragraphs, for the sake of clarity, the parameter $u_{0}$ is omitted. For instance $\vec{\beta}\left(u_{0}\right)$ is now written as $\vec{\beta}$. Thus, when no parameter is specified, $u=u_{0}$.

\subsection{Resolution parameters}

Equation (4) expresses that the spatial performances of a Radar by the product of two functions $g$ and $M$. In monostatic case, properties of $g$ gives the range resolution and examination of $M$ leads to the azimuth resolution. We extend these names to the bistatic case.

In the following, we consider several numerical examples to explain our method. These examples are detailed in section 6 . 


\subsubsection{Range resolution}

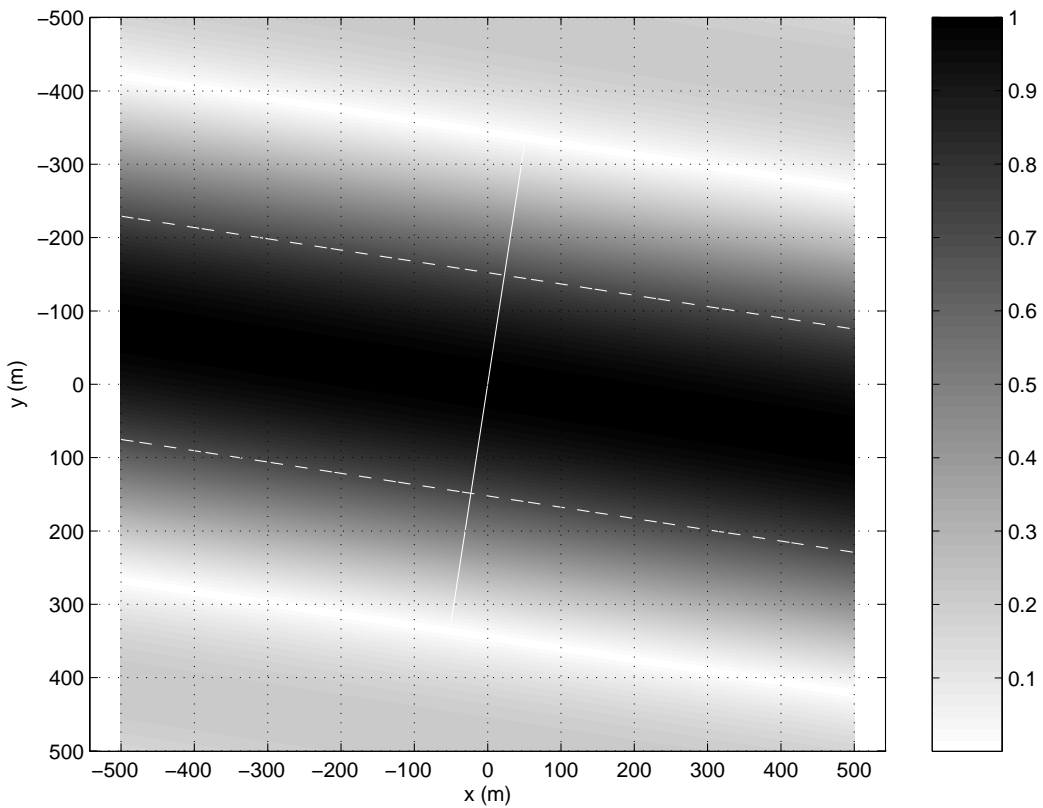

Figure 3: Map of normalized $|g|$ function in the $(x, y)$ plane, for $B=1 \mathrm{MHz}$ and $\vec{\beta}=0.134 \hat{x}-$ $0.873 \hat{y}+0.983 \hat{z}$ (i.e. for the satellite B3 with the experimental configuration described afterward). Dashed lines give the contour line at $1 / \sqrt{2}$, solid line indicates the $\hat{\beta}_{g}$ direction.

Function $g$ gives the Radar spatial performance in the range domain. The designation "range" is chosen to be consistent with the monostatic case. It describes the effect of the signal bandwidth on the resolution parameter. Starting with (6) and supposing the Radar geometry well known, map of $\left|\frac{g}{\max (g)}\right|$ is generated as a function of $x$ and $y$ (Figure 3). From figure 3, it can be noted that the ground range resolution is not defined in the orthogonal direction of $\hat{\beta}_{g}$. It is minimum when vectors $\vec{r}$ and $\hat{\beta}_{g}$ are collinear. In this way, range resolution $\delta_{r}$ is defined as a measure of the target resolvability in the range domain by a given Radar signal $g(t)$, when $\vec{r}$ is pointing along $\hat{\beta}_{g}$. In this situation, we have:

$$
\vec{r}_{\text {min }}=\frac{\delta_{r}}{2} \hat{\beta}_{g}
$$

Substituting (5 and 9) into (6) to solve for $\delta_{r}$ :

$$
\frac{\sin \left(\frac{\pi B}{c}\|\vec{\beta}\| \cos \alpha \frac{\delta_{r}}{2}\right)}{\frac{\pi B}{c}\|\vec{\beta}\| \cos \alpha \frac{\delta_{r}}{2}}=\frac{1}{\sqrt{2}}
$$

it turns out: 


$$
\delta_{r}=\frac{0.886 c}{2 B \cos \left(\frac{\beta}{2}\right) \cos \alpha}
$$

As equation (11) shows, in a bistatic Radar system, $\delta_{r}$ varies with angle $\beta$. Hence, the range resolution depends on the target position relatively to the transmitter and receiver locations. Thus, when the transmitter, the target and the receiver are strung together (forward scattering), $\beta=\pi$ and range resolution disappears. The range resolution is maximum when $\beta=0$, i.e. in the monostatic configuration.

In our example (figure 3), the length of the solid line segment is $\delta_{r}=301 \mathrm{~m}$.

\subsubsection{Azimuth resolution}

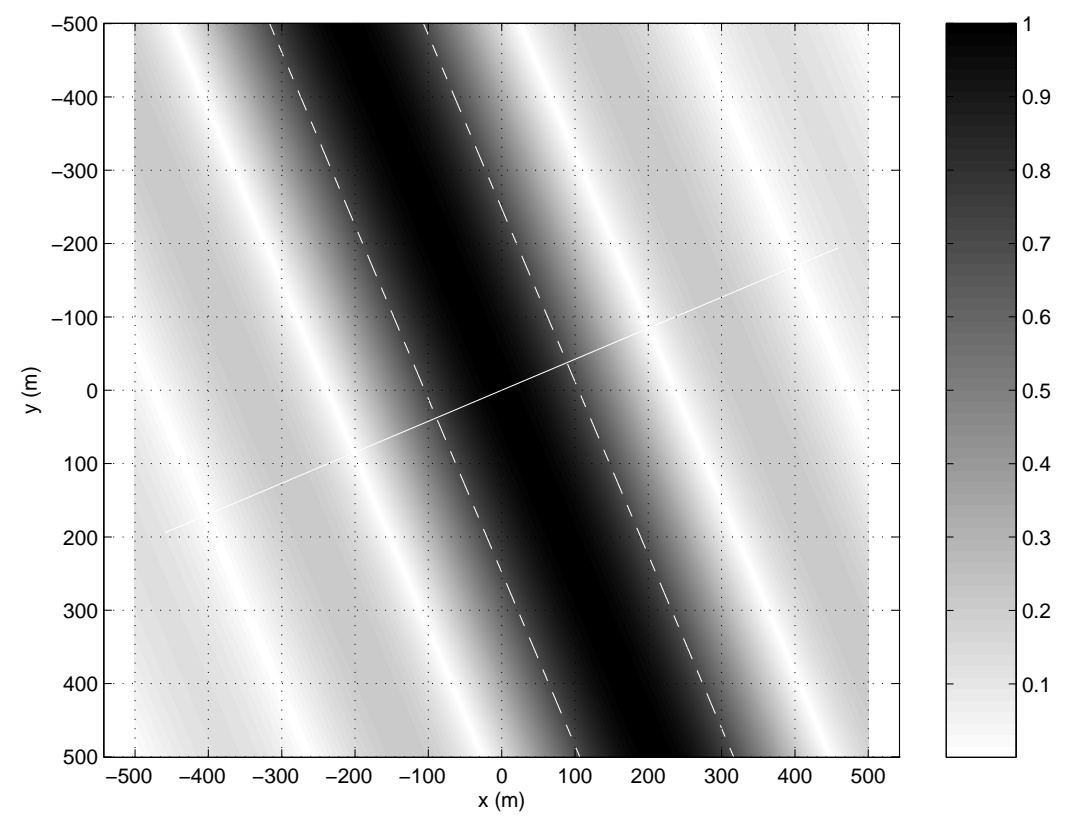

Figure 4: Map of normalized $|M|$ function in the $(x, y)$ plane, for $T=6 \mathrm{~s}, f_{0}=1575.42 \mathrm{MHz}$ and $d \overrightarrow{\beta_{g}} / d u=1.10^{-4}(-1.34 \hat{x}+0.567 \hat{y}+0.11 \hat{z})$ (i.e. for the satellite B3 with the experimental configuration described afterward). Dashed lines give the contour line at $1 / \sqrt{2}$, solid line indicates the $d \hat{\beta}_{g} / d u$ direction.

The azimuth resolution $\delta_{a}$ is expressed from the function $M$. Figure 4 shows the plot of $M$ in the ground plane. The azimuth resolution $\delta_{a}$ is defined in the direction of the projection of vector $\frac{d \vec{\beta}}{d u}$ on the plane $(x, y)$. It is given by (appendix 9.2):

$$
\delta_{a}=\frac{0.886 \lambda_{0}}{T \sqrt{\left(\frac{d \beta}{d u} \sin \left(\frac{\beta}{2}\right) \cos \alpha+2 \frac{d \alpha}{d u} \cos \left(\frac{\beta}{2}\right) \sin \alpha\right)^{2}+\left(2 \frac{d \gamma}{d u} \cos \left(\frac{\beta}{2}\right) \cos \alpha\right)^{2}}}
$$


As above, the azimuth resolution varies with the geometric configurations. In our example (figure 4), equation (12) gives $\delta_{a}=193 \mathrm{~m}$.

In a 2D bistatic Radar system, equation (12) reduces to:

$$
\delta_{a}=\frac{0.886 \lambda_{0}}{T \sqrt{\left(\frac{d \beta}{d u} \sin \left(\frac{\beta}{2}\right)\right)^{2}+\left(2 \frac{d \gamma}{d u} \cos \left(\frac{\beta}{2}\right)\right)^{2}}}
$$

\section{Interpretation}

In order to better understand equation (13), let us consider the 2D tomography system :

$$
\begin{aligned}
& \beta(u)=\theta_{e}(u)-\theta_{r}(u) \\
& \gamma(u)=\frac{\theta_{e}(u)+\theta_{r}(u)}{2}
\end{aligned}
$$

In (14), $\theta_{e}$ is the angular location of the transmitter and $\theta_{r}$ the orientation of the receiver. Assuming the equality of the receiver and the transmitter velocities (i.e. $\frac{d \theta_{e}}{d u}=\frac{d \theta_{r}}{d u}$ ), equation (13) becomes:

$$
\delta_{a}=\frac{0.886 \lambda_{0}}{2 T\left|\frac{d \theta}{d u}\right| \cos \frac{\beta}{2}}
$$

In the monostatic case $(\beta=0)$, ground azimuth resolution $\delta_{a}$ is minimal. It increases with the bistatic angle. Now, let us assume that the receiver and the transmitter velocities magnitude are equal but in the opposite direction (i.e. $\frac{d \theta_{e}}{d u}=-\frac{d \theta_{r}}{d u}$ ), (13) becomes:

$$
\delta_{a}=\frac{0.886 \lambda_{0}}{2 T\left|\frac{d \theta}{d u}\right|\left|\sin \frac{\beta}{2}\right|}
$$

Hence, $\delta_{a}$ is minimal when the receiver and the transmitter are strung together $(\beta=\pi)$ and maximal in the monostatic case. This basic example shows that the azimuth resolution depends on the bistatic angle and on the sensor velocities. 


\subsubsection{Resolution ellipse}

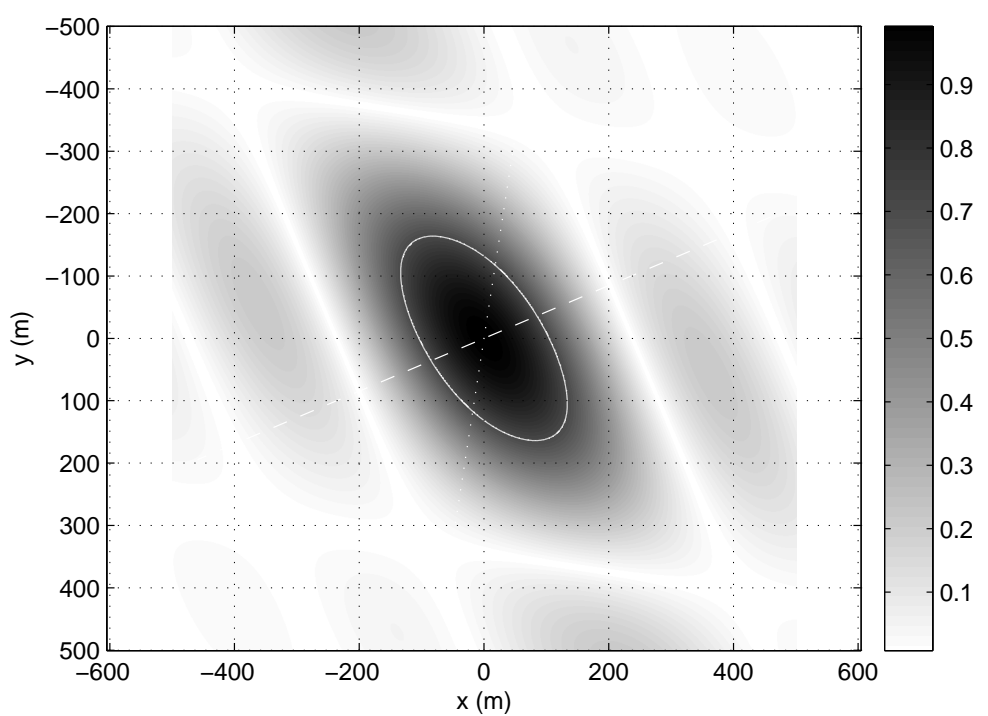

Figure 5: Map of normalized product function $|M g|$ in the $(x, y)$ plane, for $T=6 \mathrm{~s}, B=$ $1 \mathrm{MHz}$ and $f_{0}=1575.42 \mathrm{MHz}$ for the satellite $\mathrm{B} 3$ (with the experimental configuration described afterward). Solid line gives the contour line at $1 / \sqrt{2}$ (i.e. resolution ellipse), dashed line indicates the $d \hat{\beta}_{g} / d u$ direction, dotted line indicates the $\hat{\beta}_{g}$ direction.

A more general way to compute the bistatic Radar system resolution $\delta_{\theta}$ in the ground plane in any arbitrary direction $\theta$ is to solve the following expression:

$$
g\left(\frac{2 \cos \frac{\beta}{2}(\hat{\beta} \cdot \vec{\delta})}{c}\right) M\left(\frac{1}{\lambda_{0}} \frac{d \vec{\beta}}{d u} \cdot \vec{\delta}\right)=\frac{P_{0} B T}{\sqrt{2}}
$$

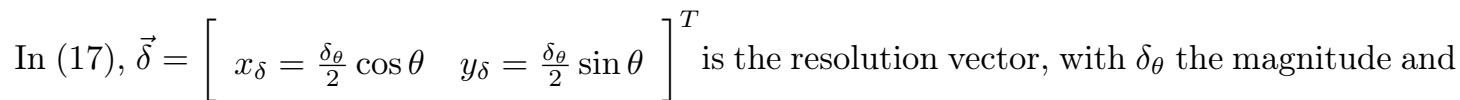
$\theta$ the direction. Parameter $\delta_{\theta}$ is a more general resolution parameter than the classical range or azimuth resolutions. It varies with: $\vec{\beta}$, the bandwidth, the central frequency of the signal and the integration time. Figure 5 shows the map of the product $\left|\frac{M g}{P_{0} B T}\right|$ in the ground plane. Dashed line represents the resolution ellipse: it is the locus of all points in the plane $(x, y)$ satisfying (17). Appendix (9.3) describes the method used to compute this resolution ellipse:

$$
\left(x_{\delta}, y_{\delta}\right)=F\left(\delta_{r}, \delta_{a}, \alpha, \gamma, \frac{d \alpha}{d u}, \frac{d \gamma}{d u}\right)
$$




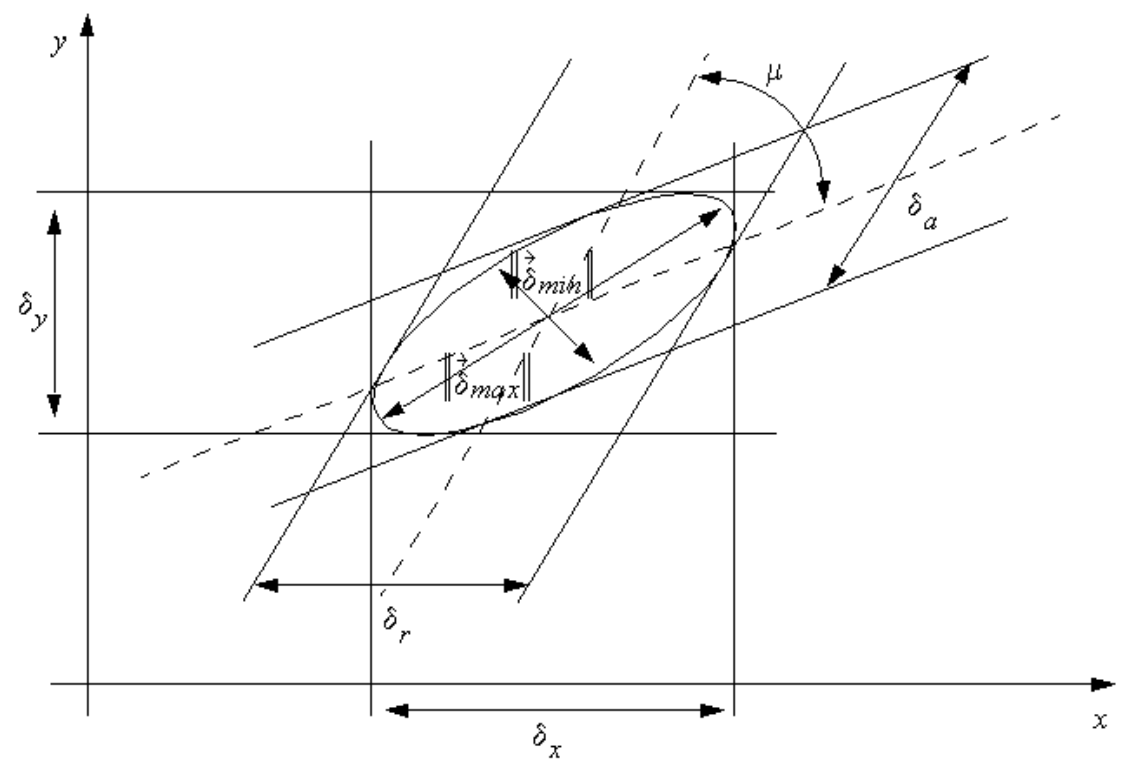

Figure 6: Resolution parameters

With equation (17), new bistatic resolution parameters are defined (figure 6):

- $\vec{\delta}_{\text {max }}=2 \max (\vec{\delta})$ describes the magnitude and orientation of the maximum value of the resolution $\delta_{\theta}$,

- $\vec{\delta}_{\text {min }}=2 \min (\vec{\delta})$ is the minimum value of $\delta_{\theta}$,

- $\delta_{x}=2\|\vec{\delta}\|_{\theta=0}$ is the resolution in the $\hat{x}$ direction; $\delta_{y}=2\|\vec{\delta}\|_{\theta=\pi / 2}$ is the resolution in the $\hat{y}$ direction,

- $A_{e}$ is the ellipse resolution area.

These parameters characterize the spatial performance of a bistatic Radar system.

Let us now introduce a new parameter $\mu$ called ambiguity angle, define as follows:

$$
\cos \mu=\frac{\vec{\beta}_{g} \cdot \frac{d \vec{\beta}_{g}}{d u}}{\left\|\vec{\beta}_{g}\right\|\left\|\frac{d \vec{\beta}_{g}}{d u}\right\|}=\frac{-\sin \left(\frac{\beta}{2}\right) \cos \alpha \frac{d \beta}{d u}-2 \cos \left(\frac{\beta}{2}\right) \frac{d \alpha}{d u} \sin \alpha}{\sqrt{\left(\frac{d \beta}{d u} \sin \left(\frac{\beta}{2}\right) \cos \alpha+2 \frac{d \alpha}{d u} \cos \left(\frac{\beta}{2}\right) \sin \alpha\right)^{2}+\left(2 \frac{d \gamma}{d u} \cos \left(\frac{\beta}{2}\right) \cos \alpha\right)^{2}}}
$$

$\mu$ gives useful information on the resolution capabilities of a bistatic Radar system. In order to study (19), let us first consider a 2D Radar system (i.e. when $\alpha=0$ ). In this case $\hat{\beta}_{g}=\hat{\beta}$ the bisector of the bistatic system. Eq. 19 becomes: 


$$
\cos \mu=\frac{-\sin \left(\frac{\beta}{2}\right) \frac{d \beta}{d u}}{\sqrt{4 \cos ^{2}\left(\frac{\beta}{2}\right)\left\|\frac{d \hat{\beta}_{g}}{d u}\right\|^{2}+\left(\frac{d \beta}{d u}\right)^{2} \sin ^{2}\left(\frac{\beta}{2}\right)}}
$$

For the monostatic case $(\beta=0)$, equation (20) gives $\cos \mu=0$, i.e. $\mu=\frac{\pi}{2}$. Then, vector $\vec{\beta}_{g}$ is perpendicular to $\frac{d \vec{\beta}_{g}}{d u}$. In this context, range and azimuth resolution parameters are pertinent: each of them gives the resolution in the two orthogonal directions $\hat{\beta}_{g}$ and $\frac{d \hat{\beta}_{g}}{d u}$, i.e. $\left(\delta_{a}, \delta_{r}\right)=$ $\left(\left\|\vec{\delta}_{\max }\right\|,\left\|\vec{\delta}_{\min }\right\|\right)$ (figure 6$)$.

In a $2 \mathrm{D}$ bistatic Radar system, the same result is achieved if $\beta$ angle remains constant during the integration time. On the other hand the normalized dot product in (20) gives -1 (i.e $\mu=\pi$ ) when angle $\beta=\pi$ or/and $\left\|\frac{d \hat{\beta}_{g}}{d u}\right\|=0$. The first case corresponds to the forward direction. In the second case, there is no variation of the vector $\hat{\beta}_{g}$ direction during the integration time.

\section{Multistatic Generalized Ambiguity Function (MGAF)}

Multistatic Radar can be seen as a set of $M_{s}$ bistatic Radar. In this way, several sets of data are available (i.e. one for each bistatic system). There are two options to perform the data fusion [21]: (i) a coherent summation between them or (ii) an incoherent summation. The coherent summation gives a multistatic GAF plot with a well-defined central response at the target position surrounded by a series of secondary peaks. These peaks may be considered similar to grating lobes in standard antenna array theory. Hence the side lobe ratio is very large, making the detection process more difficult. For the incoherent summation, multistatic GAF plot has only one larger peaks centered at the target location. Indeed, since only the magnitude response of each bistatic Radar is summed, the array beam forming effect does not exist.

In this paper, the performances of a MAMF Radar system are only determined by the evaluation of the multistatic incoherent GAF:

$$
\chi_{i n c}(\vec{r})=\sum_{n=1}^{M_{s}} g_{n} M_{n}=\sum_{n=1}^{M_{s}} P_{n} B_{n} T_{n}\left|\frac{\sin \left(\frac{\pi B_{n}}{c} \overrightarrow{\beta_{n}} \cdot \vec{r}\right)}{\frac{\pi B_{n}}{c} \vec{\beta}_{n} \cdot \vec{r}}\right|\left|\frac{\sin \left(\frac{k_{n} T_{n}}{2} \frac{d \overrightarrow{\beta_{n}}}{d u} \cdot \vec{r}\right)}{\frac{k_{n} T_{n}}{2} \frac{d \overrightarrow{\beta_{n}}}{d u} \cdot \vec{r}}\right|
$$

In $(21), M_{s}$ is the total number of transmitter-receiver pairs and $k_{n}=2 \pi f_{0 n} / c$ is the wave number for the considered bistatic system. $f_{0 n}$ is the carrier frequency of the transmitted signal, $B_{n}$ its bandwidth and $P_{n}$ its power. $T_{n}$ is the integration time for the considered transmitterreceiver pair.

As in the bistatic Radar system case, performances for a MAMF Radar system are given by $\vec{\delta}_{\text {max }}, \vec{\delta}_{\text {min }}, \delta_{x}, \delta_{y}$ and $A_{e}$. These parameters are derived from equation (22): 


$$
\sum_{n=1}^{M_{s}}\left|\frac{\sin \left(\frac{\pi B_{n}}{c} \vec{\beta}_{n} \cdot \vec{r}\right)}{\frac{\pi B_{n}}{c} \vec{\beta}_{n} \cdot \vec{r}}\right|\left|\frac{\sin \left(\frac{k_{n} T_{n}}{2} \frac{d \overrightarrow{\beta_{n}}}{d u} \cdot \vec{r}\right)}{\frac{k_{n} T_{n}}{2} \frac{d \overrightarrow{\beta_{n}}}{d u} \cdot \vec{r}}\right|=\frac{M_{s}}{\sqrt{2}}
$$

\section{Synthesis}

Table 1: Pertinence of the resolution parameters

\begin{tabular}{|c|c|c|c|c|c|c|c|}
\hline$\delta_{r}$ & $\delta_{a}$ & $\vec{\delta}_{\max }$ & $\vec{\delta}_{\min }$ & $\delta_{x}$ & $\delta_{y}$ & $\mu$ & $A_{e}$ \\
\hline \hline- & - & +++ & +++ & + & + & +++ & ++ \\
\hline
\end{tabular}

A summary of our results is given in Table 1. The pertinence of the bistatic and multistatic resolution parameters are described above. Range and azimuth resolutions $\delta_{r}$ and $\delta_{a}$ are the less valuables: the range resolution $\delta_{r}$ is not only a function of the bandwidth of the transmitted signal but depends on $\vec{\beta}$. Similarly the azimuth resolution $\delta_{a}$ is not only a function of the carrier frequency and of the integration time, but also a function of the Radar geometry. $\delta_{r}$ and $\delta_{a}$ describe the effect of the bandwidth and the integration time but not directly the capacity of the Radar system to separate two close targets. Therefore, several new parameters have been introduced:

- Parameter $\vec{\delta}_{\text {max }}$ defined as the minimum distance between two close targets detected by the Radar system. $\vec{\delta}_{\text {max }}$ is characterized by its magnitude and orientation (in respect with the $x$ axis). It expresses the lowest length and establishes the upper bound of the resolution.

- Parameter $\vec{\delta}_{\min }$ evaluates the lower bound.

- $\delta_{x}$ and $\delta_{y}$ are the magnitude of $\vec{\delta}$ evaluated on the $\mathrm{x}$ and $\mathrm{y}$ axis.

- The ellipse area $A_{e}$ describes the detection area (i.e. the surface described by equation $18)$.

- The parameter $\mu$ is used to illustrate the capability of a bistatic Radar system to give a good resolution. It can be used in order to introduce an additional constraint in the choice of sensor configurations for a MAMF experiment.

All the resolution parameters are space dependent. As the illuminated area is small, the resolution parameters are given for a target located at the center of the studied area. 


\section{Validation}

Bistatic/multistatic Radar system allows the use of transmitters of opportunity, such as GNSS satellites. Now to illustrate our work, we characterize the spatial resolution on a bistatic/multistatic Radar system based on GPS satellites.

\subsection{System overview}

The studied MAMF Radar system is built with GPS satellites as non-cooperative transmitters [22] and with one motionless receiver localized on the ground (figure 7). The center of the scene to be imaged is located at the origin of coordinates and the ground plane is the $(x, y)$ plane. The satellite location at time $u$ is given by $\vec{L}_{E}(u)$. Similarly, the receiver location is $\vec{L}_{R}$. GPS satellites transmit two low power radio signals, called L1 and L2. We use the open GPS signal L1 with a frequency $f_{0}=1575.42 \mathrm{MHz}$. L1 is Bipolar-Phase Shift Key (BPSK) modulated with a Pseudo Random Noise (PRN) 1.023MHz code known as the Coarse/Acquisition (C/A) code. For sake of clarity, we assume that transmitted pulses have uniform power over the frequency range: $f \in\left[f_{0}-B / 2, f_{0}+B / 2\right]$ with $B=1 \mathrm{MHz}$. The target is assumed located on the ground near the town of Saint Emilion (small city near Bordeaux - France - North $45^{\circ}$, East $0^{\circ}$ ). During the integration time $(u \in[-T / 2, T / 2])$, the target is seen simultaneously by 3 or 4 GPS satellites (figure 8). In this work, satellites called 'A1', 'B3' and 'F1' are used as transmitters. The elevation cut-off is equal to $45^{\circ}$ (i.e. we exclude satellites below that elevation).

The MAMF Radar system parameters are listed in table 2. Note that in all cases the antenna gain patterns (transmitters and receivers) are considered isotropic.

Table 2: MAMF Radar system parameters

\begin{tabular}{|c|c|c|}
\hline Parameters & Symbol & Values \\
\hline \hline Carrier frequency & $f_{0}$ & $1575.42 \mathrm{MHz}$ \\
\hline Transmitted signal bandwidth & $B$ & $1 \mathrm{MHz}$ \\
\hline Integration time & $T$ & $60 \mathrm{~s}$ \\
\hline Receiver position & $\left(x_{r}, y_{r}\right)$ & $(0,-2000) \mathrm{m}$ \\
\hline
\end{tabular}




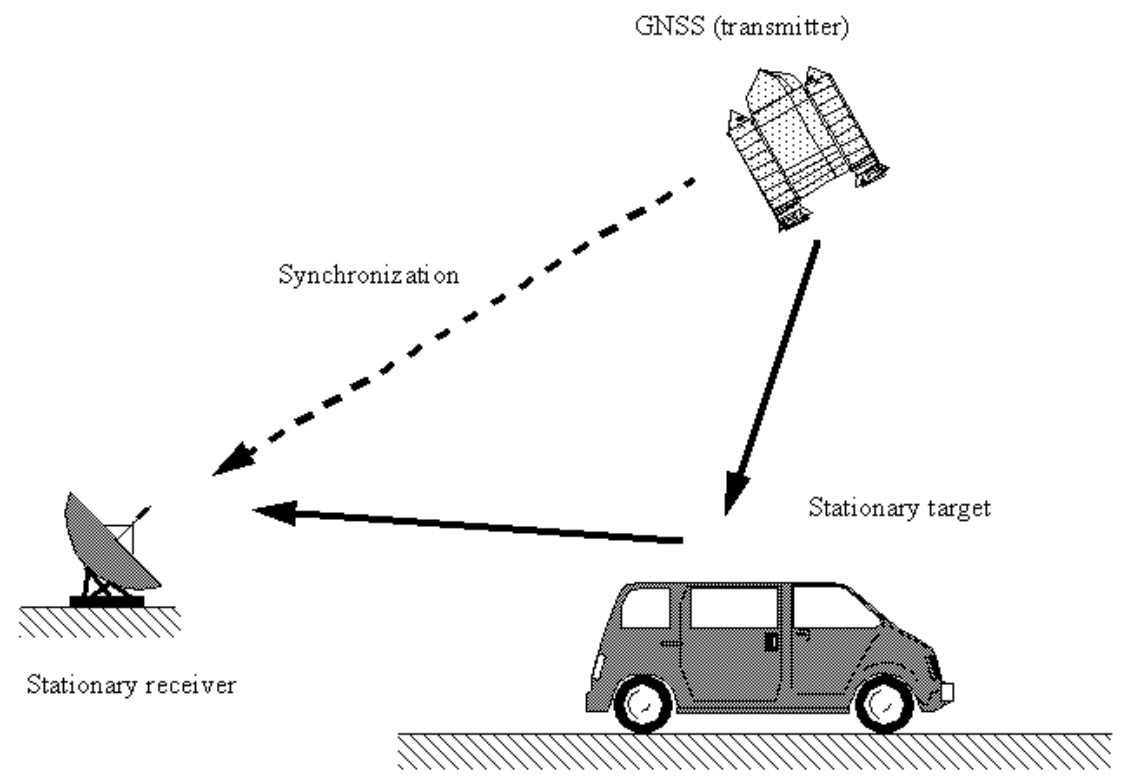

Figure 7: MAMF Radar system geometry

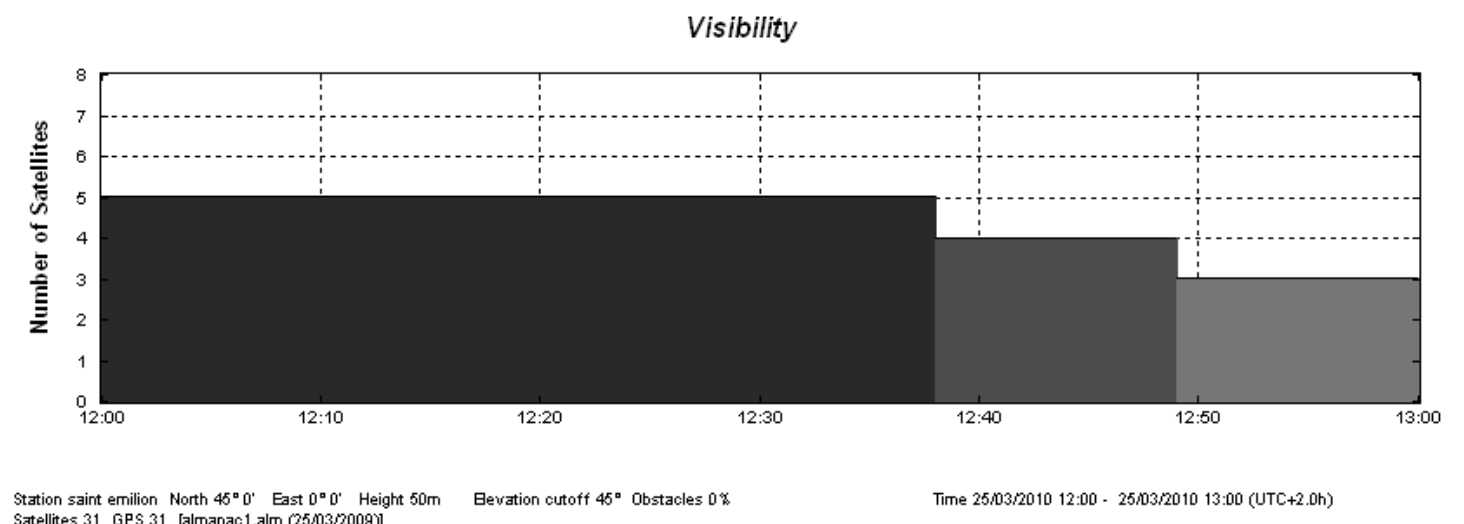

Figure 8: Satellites visibility [22]. Elevation cut-off equal $45^{\circ}$

Table 3 shows the change of the azimuth and elevation angles of A1, B3 and F1 transmitters during the integration time. For instance, the satellite A1 has a mean azimuth angle equal to $147^{\circ}$ with $1.45^{\circ}$ of angular variation and a mean elevation angle of $74^{\circ}$ with $0.25^{\circ}$ of change. These very small changes observed during $T$, set a poor resolution capability.

Table 3: Transmitter location as a function of integration time - $T=60 \mathrm{~s}$

\begin{tabular}{|c|c|c|c|c|}
\hline Sat. & $\theta_{e}\left(u_{0}\right)$ & $\varphi_{e}\left(u_{0}\right)$ & $\Delta \theta_{e}$ & $\Delta \varphi_{e}$ \\
\hline \hline$A_{1}$ & $74^{\circ}$ & $147^{\circ}$ & $0.248^{\circ}$ & $1.45^{\circ}$ \\
\hline$B_{3}$ & $79.3^{\circ}$ & $43.4^{\circ}$ & $0.204^{\circ}$ & $2.48^{\circ}$ \\
\hline$F_{1}$ & $78.9^{\circ}$ & $265^{\circ}$ & $0.284^{\circ}$ & $2.06^{\circ}$ \\
\hline
\end{tabular}




\subsection{Point Spread Function (PSF)}

The system impulse response (the Point Spread Function) is computed to evaluate resolution parameters and to validate the results obtained with the GAF computation. The target, a single point target reflector, is localized at the origin of coordinates. With these assumptions, the PSF function is given by:

$$
\operatorname{PSF}(\vec{r})=\int_{u_{0}-T / 2}^{u_{0}+T / 2} \int_{f_{0}-B / 2}^{f_{0}+B / 2} \exp \left(-j \frac{2 \pi f}{c} \vec{\beta}(u) \cdot \vec{r}\right) d f d u
$$

(23) is computed as follow: first, a regular grid of spatial frequency $\left(k_{x}, k_{y}\right)$ of the image plane is generated. This grid is determined by the extension of the image plane $\left(2 X_{\max }\right.$ by $\left.2 Y_{\max }\right)$ :

$$
\begin{aligned}
& k_{x}(i)=\frac{-\pi}{\Delta_{x}}+i \frac{\pi}{X_{\max }} \\
& k_{y}(i)=\frac{-\pi}{\Delta_{y}}+i \frac{\pi}{Y_{\max }}
\end{aligned}
$$

In $(24), \Delta_{x, y}$ are the pixel size.

Secondly, for each measurement data, i.e. for each pair $(f, u)$, the experimental spatial frequency components are computed:

$$
\begin{aligned}
& k_{x m}(f, u)=\frac{2 \pi f}{c} \vec{\beta}(u) \cdot \hat{x} \\
& k_{y m}(f, u)=\frac{2 \pi f}{c} \vec{\beta}(u) \cdot \hat{y}
\end{aligned}
$$

In $(25) k_{x m}$ and $k_{y m}$ are not uniformly spaced to use a FFT. So a neighborhood interpolation is applied to create a regular mapping between the measurement plane $\left(k_{x m}, k_{y m}\right)$ and the image plane $\left(k_{x}, k_{y}\right)$. Unitary amplitude stands on this corresponding frequency place. Then (23) is computed through two fast Fourier transforms. This method is based on the classical Polar Format Algorithm, used in many monostatic Radar system [23]. Finally, the resolution ellipse is computed:

$$
\operatorname{PSF}(\vec{r})=\frac{1}{\sqrt{2}}
$$

\subsection{Simulation results}

In this part, PSF and GAF are computed and compared. First the bistatic scenarios are studied, then the multistatic case is considered. 


\subsubsection{Bistatic scenarios}

Table 4 gives bistatic resolution parameters computed with (18):

- The poorest resolution capability (i.e. $20.8 \mathrm{~m} \leq \delta_{\theta} \leq 1340 \mathrm{~m}$ ) is obtained with the satellite A1. This is mainly due to the A1 satellite location. This effect is highlighted with the ambiguity angle $\mu=13^{\circ}$.

- The range and azimuth resolutions are not relevant to define the capability of a Radar. Indeed, although range resolution obtained with satellites A1 and B3 are similar, the spatial performances achieved with B3 satellite are much better than those achieved with satellite A1.

- In all the three cases, the resolution capability is poor and not efficient to detect two close targets with an integration time of $60 \mathrm{~s}$.

Table 4: Bistatic resolution parameters $-\mathrm{T}=60 \mathrm{~s}-(\mathrm{x}, \mathrm{y})$ plane

\begin{tabular}{|c|c|c|c|c||c|c|c|c|}
\hline $\begin{array}{c}\text { Sat. } \\
\text { ref. }\end{array}$ & $\begin{array}{c}\delta_{r} \\
(\mathrm{~m})\end{array}$ & $\begin{array}{c}\delta_{a} \\
(\mathrm{~m})\end{array}$ & $\begin{array}{c}\| \vec{\delta}_{\text {max }} \\
(\mathrm{m})\end{array}$ & $\begin{array}{c}\left\|\vec{\delta}_{\text {min }}\right\| \\
(\mathrm{m})\end{array}$ & $\begin{array}{c}\delta_{x} \\
(\mathrm{~m})\end{array}$ & $\begin{array}{c}\delta_{y} \\
(\mathrm{~m})\end{array}$ & $\begin{array}{c}\mu \\
\left({ }^{\circ}\right)\end{array}$ & $\begin{array}{c}A_{e} \\
\left(\mathrm{~m}^{2}\right)\end{array}$ \\
\hline \hline A1 & 302 & 20.8 & 1340 & 20.8 & 513 & 20.8 & 13 & $2.210^{4}$ \\
\hline B3 & 301 & 19.3 & 354 & 19.3 & 20.4 & 49.9 & 58.3 & $5.3610^{3}$ \\
\hline F1 & 223 & 19.9 & 258 & 19.9 & 23.7 & 38.7 & 59.6 & $4.0310^{3}$ \\
\hline
\end{tabular}

Figures (9, 10 and 11) show the map of (23) in the ground plane. The dotted line, is the resolution ellipses computed with (18) and the solid one is the ellipses determined with (26). In all cases, the same orientation of the ellipse is found with both methods. Only small differences are observed on the magnitude. This is due to the assumptions used to calculate (4). Results are presented on table (5).

Table 5: Resolutions parameters computed with (18) and (23) (magnitude, direction )

\begin{tabular}{|c|c|c|c|c|}
\hline $\begin{array}{c}\text { Sat. } \\
\text { ref. }\end{array}$ & $\begin{array}{c}\vec{\delta}_{\max } \\
(18)\end{array}$ & $\begin{array}{c}\vec{\delta}_{\max } \\
(26)\end{array}$ & $\begin{array}{c}\vec{\delta}_{\min } \\
(18)\end{array}$ & $\begin{array}{c}\vec{\delta}_{\min } \\
(26)\end{array}$ \\
\hline \hline $\mathrm{A} 1$ & $\left(1340 \mathrm{~m}, 178^{\circ}\right)$ & $\left(1278 \mathrm{~m}, 178^{\circ}\right)$ & $\left(20.8 \mathrm{~m}, 272^{\circ}\right)$ & $\left(20.4 \mathrm{~m}, 270^{\circ}\right)$ \\
\hline $\mathrm{B} 3$ & $\left(354 \mathrm{~m}, 247^{\circ}\right)$ & $\left(292 \mathrm{~m}, 247^{\circ}\right)$ & $\left(19.3 \mathrm{~m}, 335^{\circ}\right)$ & $\left(19 \mathrm{~m}, 335^{\circ}\right)$ \\
\hline F1 & $\left(258 \mathrm{~m}, 120^{\circ}\right)$ & $\left(221 \mathrm{~m}, 120^{\circ}\right)$ & $\left(19.9 \mathrm{~m}, 29.5^{\circ}\right)$ & $\left(19.6 \mathrm{~m}, 31^{\circ}\right)$ \\
\hline
\end{tabular}

In terms of resolution performances, the three bistatic systems presented here are quite different (figure 9, 10 and 11). Bistatic radar with satellite A1 has the poorest resolution capability. Satellites B3 and F1 provide similar resolution parameters but the orientations of their resolution ellipses are different. This analysis gives good insight for the use of these satellites in a MAMF Radar system. 


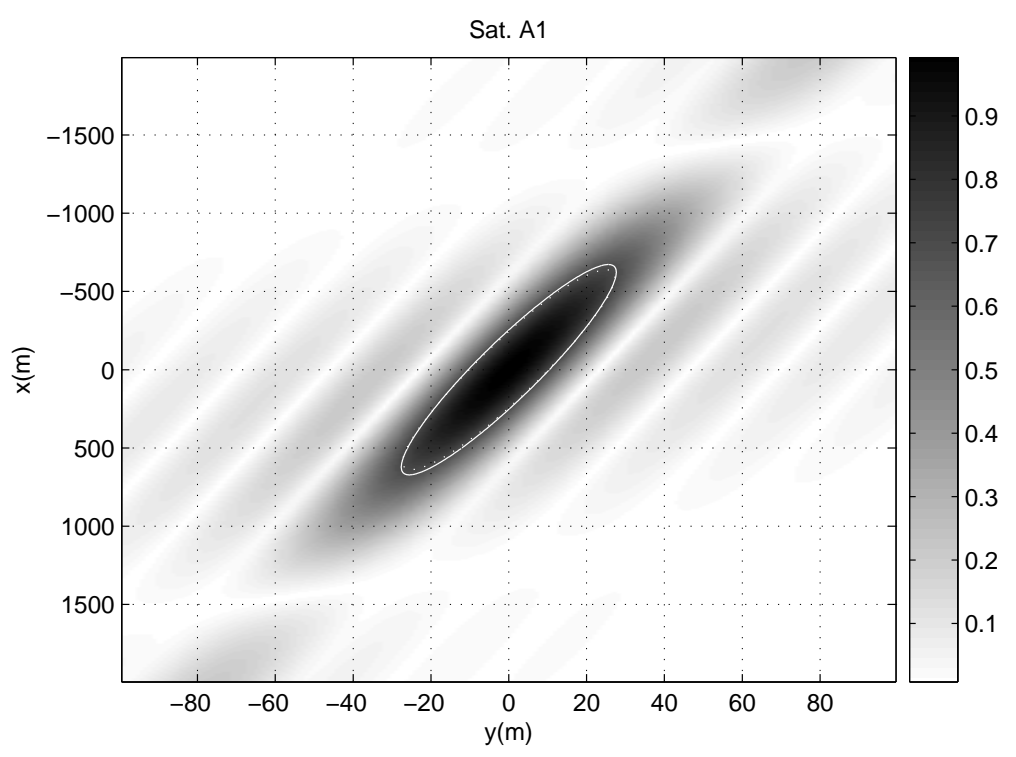

Figure 9: Map of PSF for sat. A1 in the ground plane. Solid line gives the resolution ellipse computed with (18). Dotted line indicates the resolution ellipse obtained with (23).

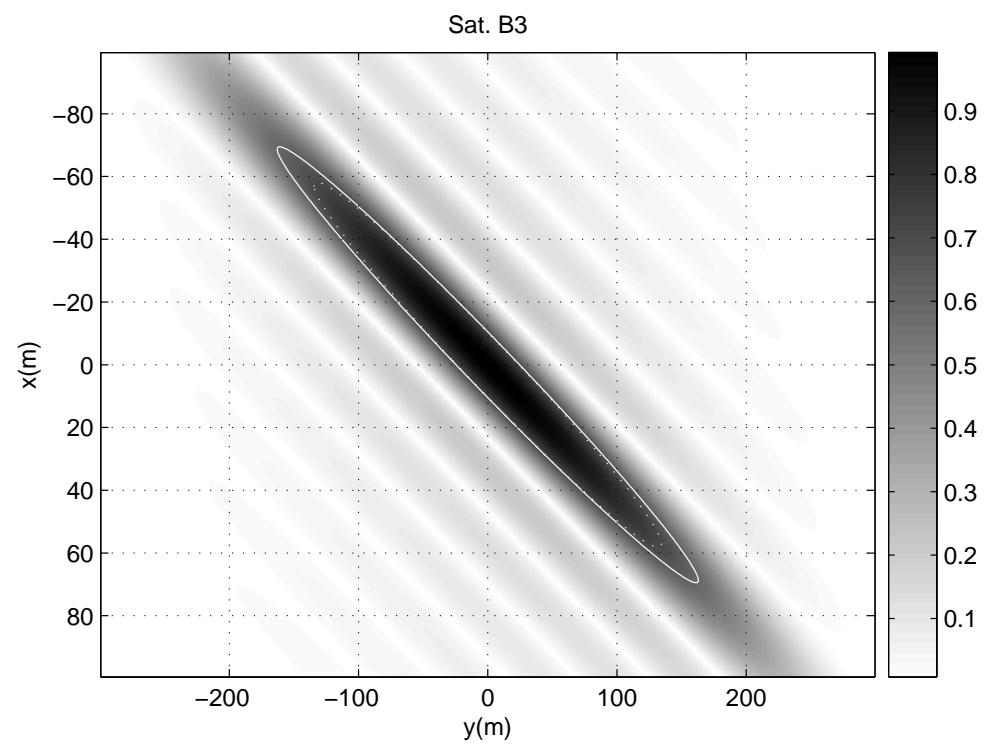

Figure 10: Map of PSF for sat. B3 in the ground plane. Solid line gives the resolution ellipse computed with (18). Dotted line indicates the resolution ellipse obtained with (23). 


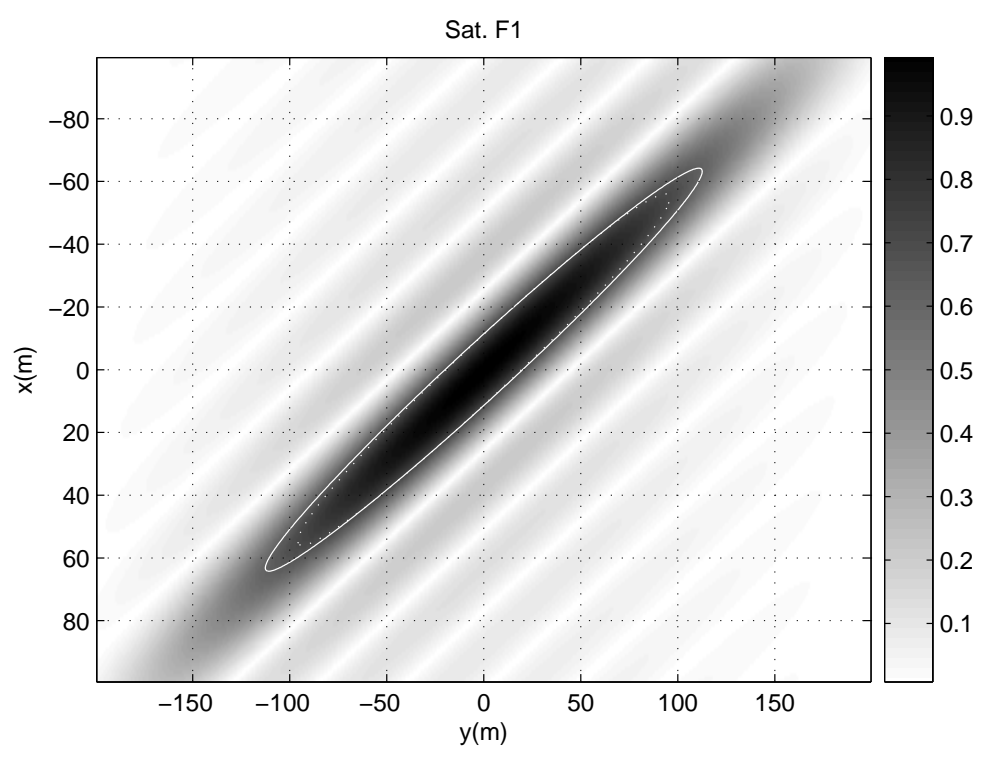

Figure 11: Map of PSF for sat. F1 in the ground plane. Solid line gives the resolution ellipse computed with (18). Dotted line indicates the resolution ellipse obtained with (23).

\subsubsection{Multistatic context}

To evaluate the performance of the MAMF Radar system built with satellites A1, B3 and F1, we compute: (i) the Multistatic GAF (M-GAF) (equations (21)) and (ii) the Multistatic PSF (equation (23)).

In table 6 are resumed the resolution parameters obtained from MGAF. In comparison with the bistatic system, the unfavorable resolution resolution parameter $\left(\left\|\vec{\delta}_{\text {max }}\right\|\right)$ is divided by 10: The multisatic system strongly improve the Radar resolution.

Table 6: Theoretical values of the multistatic resolution parameters - $\mathrm{T}=60 \mathrm{~s}-(\mathrm{x}, \mathrm{y})$ plane

\begin{tabular}{|c|c|c|c|c|}
\hline$\vec{\delta}_{\text {max }}\left(\mathrm{m},{ }^{\circ}\right)$ & $\vec{\delta}_{\min }\left(\mathrm{m},{ }^{\circ}\right)$ & $\delta_{x}(\mathrm{~m})$ & $\delta_{y}(\mathrm{~m})$ & $A_{e}\left(\mathrm{~m}^{2}\right)$ \\
\hline \hline$\left(31.2 \mathrm{~m}, 101^{\circ}\right)$ & $\left(27.2 \mathrm{~m}, 19^{\circ}\right)$ & 27.6 & 31 & 666 \\
\hline
\end{tabular}

Table 7 and figure 12 show comparison between MGAF and MPSF. A very small difference is visible on $\left\|\vec{\delta}_{\max }\right\|$ but the error is negligible (less than $1.5 \%$ ).

Table 7: Resolutions parameters computed with MPSF and MGAF (magnitude, direction )

\begin{tabular}{|c|c|c|c|}
\hline \multicolumn{2}{|c|}{$\vec{\delta}_{\max }$} & \multicolumn{2}{c|}{$\vec{\delta}_{\min }$} \\
\hline MGAF & MPSF & MGAF & MPSF \\
\hline \hline$\left(31.2 \mathrm{~m}, 101^{\circ}\right)$ & $\left(30.8 \mathrm{~m}, 101^{\circ}\right)$ & $\left(27.2 \mathrm{~m}, 19^{\circ}\right)$ & $\left(27.1 \mathrm{~m}, 17^{\circ}\right)$ \\
\hline
\end{tabular}

The multistatic ambiguity shape represents an intersection of the bistatic ellipsoids. Each bistatic ellipsoid is a function of the positions of the target, the transmitter and receiver. The resolution 
ellipse for each bistatic link can be computed (eq 18). Resolution parameters for the multistatic system are evaluated from the shape of the intersection of the resolution ellipses. They are calculated by a graphical method or by a numerical approach. Here, the transmitted signal for all the transmitters have the same properties. In the same way, the satellites velocities are equivalent. With this example, parameters $\left|\vec{\delta}_{\text {min }}\right|$ for all the bistatic links are approximately identical. As result, the multistatic ambiguity shape is near a circle with a radius given by $\left|\vec{\delta}_{\text {min }}\right|$.

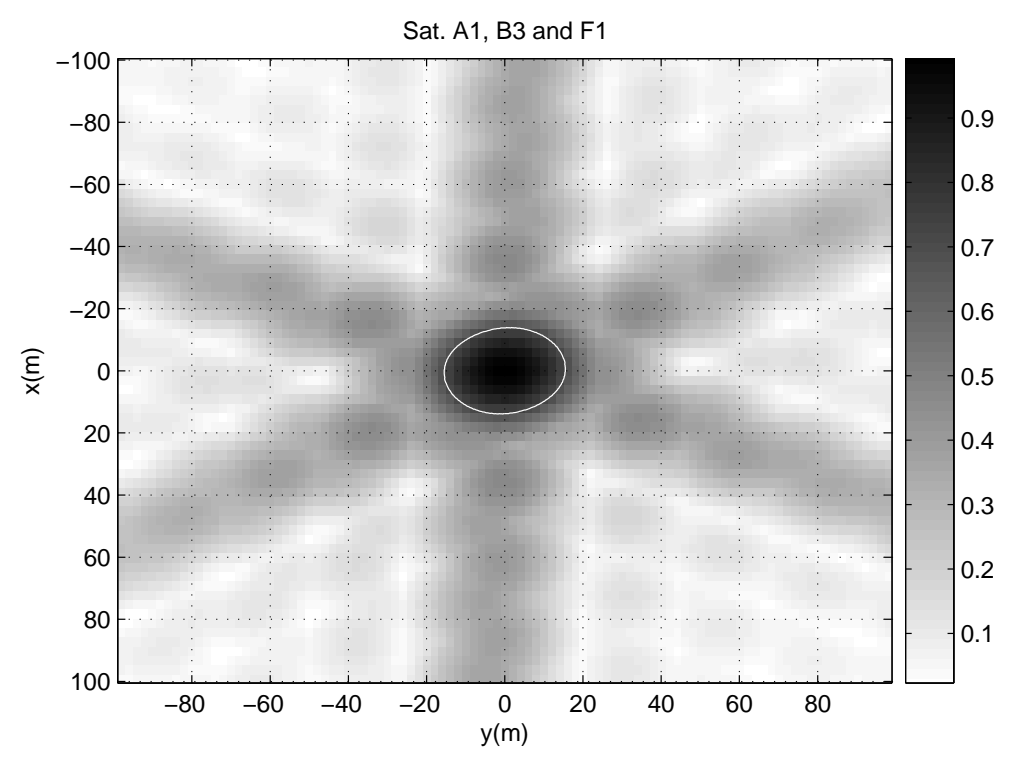

Figure 12: Map of MPSF on the ground plane. Integration time: 60s. Solid line gives the resolution ellipse computed with MGAF.

\section{Conclusion}

In this paper, the performance of bistatic/multistatic Radar system are investigated. Two methods are presented: one is a theoretical study of the Generalized Ambiguity Function (GAF); the second is the numerical study of the Point Spread Function (PSF). In the first case, our main concern is to establish analytical forms for the range and azimuth resolutions in a bistatic Radar system configuration. We point out that range and azimuth resolution are not the most relevant parameters to describe the spatial performance of the bistatic Radar: they do not evaluate the capability of the Radar system to separate two close targets. In this way, we define a new set of resolution parameters:

- $\vec{\delta}_{\text {max }}$ (magnitude and orientation) describes the minimum distance between two close targets detected by the Radar. It expresses the upper bound of the resolution, i.e. the poorest case. 
- In the same way $\vec{\delta}_{\text {min }}$ gives the lower bound.

- The multistatic ambiguity shape represents the intersection of the bistatic ellipsoids. Each bistatic ellipsoid is a function of the positions of the target, of the transmitter and of the receiver. The resolution ellipse for each bistatic link is computed. Resolution parameters for the multistatic system are evaluated from the shape of the intersection of the resolution ellipses. They are calculated by a graphical method or by a numerical approach. With our example, the transmitted signal for all the transmitters have the same properties. In

the same way, the satellites velocities are equivalent. Then, parameters $\left|\vec{\delta}_{\text {min }}\right|$ for all the bistatic links are approximately identical. As result, the multistatic ambiguity shape is near a circle with a radius given by $\left|\vec{\delta}_{\text {min }}\right|$.

- A more general way to study Radar performance, is to evaluate the resolution $\delta_{\theta}$ for a given orientation $\theta$. It is computed through the evaluation of the amplitude of the GAF and gives the resolution ellipse.

- New other parameters are also introduced, as the ambiguity angle $\mu$, the ellipse area and parameters $\delta_{x}, \delta_{y}$.

The resolution performances of a Radar system are also computed with the Point Spread Function. They are evaluated by using the Polar Format Algorithm. Comparison with the GAF shows very good agreement and allows to validate the theoretical approach. We show also, that resolution parameters may be improved by considering multiple transmitters.

\section{ACKNOWLEDGMENTS}

Funding was provided by the PEA DTC-program and the French Aerospace Lab. (ONERA).

\section{APPENDIXES}

\subsection{GAF computation}

In this part, the GAF computation is presented; we start with the following equation (27):

$$
\chi(\vec{A}, \vec{B})=K \int s_{A}(t, u) s_{B}^{*}(t, u) d t d u
$$

where $s_{A}(t, u)$ and $s_{B}(t, u)$ are the demodulated measured signals each returned by a point target with unit Radar cross section at points A and B: 


$$
\begin{aligned}
& s_{A}(t, u)=p\left(t-\frac{R_{A}}{c}\right) \exp \left(-j 2 \pi f_{0} t\right) \\
& s_{B}(t, u)=p\left(t-\frac{R_{B}}{c}\right) \exp \left(-j 2 \pi f_{0} t\right)
\end{aligned}
$$

In (28), $f_{0}$ is the carrier frequency and $R_{A}$ and $R_{B}$ the distances between the transmitter, the target and the receiver. They are given by:

$$
\begin{aligned}
& R_{A} \simeq L_{R}+L_{E}-\vec{A} \cdot \vec{\beta}(u) \\
& R_{B} \simeq L_{R}+L_{E}-\vec{B} \cdot \vec{\beta}(u)
\end{aligned}
$$

We use the following assumptions:

$$
\left\{\begin{aligned}
\frac{\|\vec{A}\|}{L_{E}} & \ll 1 \\
\frac{\|\vec{A}\|}{L_{R}} & \ll 1 \\
\frac{\|\vec{B}\|}{L_{E}} & \ll 1 \\
\frac{\|\vec{B}\|}{L_{R}} & \ll 1
\end{aligned}\right.
$$

In the frequency domain, equation (27) becomes:

$$
\chi(\vec{r})=K \iint\left|P\left(f-f_{0}\right)\right|^{2} \exp (-j k \vec{r} \cdot \vec{\beta}) \exp \left(j k_{0} \vec{r} \cdot \vec{\beta}\right) d f d u
$$

with $\vec{r}=\vec{A}-\vec{B}$.

Vector $\vec{\beta}$ is approximated by its first order Taylor expansion at $u=u_{0}$, the integration time at midpoint:

$$
\vec{\beta} \simeq \vec{\beta}\left(u_{0}\right)+\left(u-u_{0}\right) \frac{d \vec{\beta}}{d u}\left(u_{0}\right)
$$

With the assumption $B<\frac{2 c}{\pi T \vec{r} \cdot \frac{d \vec{\beta}}{d u}\left(u_{0}\right)}$, i.e the transmitted signal is narrow band, equation (31) becomes:

$$
\chi(\vec{r})=K_{1} \int_{-B / 2}^{B / 2}\left|P\left(f-f_{0}\right)\right|^{2} \exp \left(-j k \vec{r} \cdot \vec{\beta}\left(u_{0}\right)\right) d f \int_{-\infty}^{\infty} m(u) \exp \left(j k_{0} u \vec{r} \cdot \frac{d \vec{\beta}}{d u}\left(u_{0}\right)\right) d u
$$

with $m(u)=1$ if $\left|u-u_{0}\right|<T / 2$ otherwise $m(u)=0$.

By using the Fourier properties, equation (33) becomes : 


$$
\chi(\vec{r})=K_{1} g\left(\frac{\vec{r} \cdot \vec{\beta}\left(u_{0}\right)}{c}\right) M\left(\frac{\vec{r} \cdot \frac{d \vec{\beta}}{d u}\left(u_{0}\right)}{\lambda_{0}}\right)
$$

with $g(t)$ the inverse Fourier transform of the signal power spectrum $\left|P\left(f-f_{0}\right)\right|^{2}$ and $M(f)$ the IFT of $m(u)$.

\subsection{Azimuth resolution}

We start with:

$$
\frac{d \vec{\beta}}{d u}=\vec{U}+U_{1} \hat{z}
$$

In (35), $\vec{U}$ is the projection of $\frac{d \vec{\beta}}{d u}$ on the $(x, y)$ plane. Ground azimuth resolution is found in the direction $\frac{\vec{U}}{\|\vec{U}\|}$, hence we set:

$$
\vec{r}=\frac{\vec{U}}{\|\vec{U}\|} \frac{\delta_{a}}{2}
$$

The dot product $\frac{d \vec{\beta}}{d u} \cdot \vec{r}$ is obtained:

$$
\frac{d \vec{\beta}}{d u} \cdot \vec{r}=\|\vec{U}\| \frac{\delta_{a}}{2}
$$

To evaluate $\|\vec{U}\|$, we write vector $\vec{\beta}$ as follows:

$$
\vec{\beta}=2 \cos \left(\frac{\beta}{2}\right)\left(\hat{\beta}_{g} \cos \alpha+\sin \alpha \hat{z}\right)
$$

with $\hat{\beta}_{g}=\cos \gamma \hat{x}+\sin \gamma \hat{y}$. After some calculations, vector $\vec{U}$ can be expressed as follows:

$$
\vec{U}=\hat{\beta}_{g}\left(-\sin \left(\frac{\beta}{2}\right) \cos \alpha \frac{d \beta}{d u}-2 \cos \left(\frac{\beta}{2}\right) \frac{d \alpha}{d u} \sin \alpha\right)+2 \cos \left(\frac{\beta}{2}\right) \cos \alpha \frac{d \hat{\beta}_{g}}{d u}
$$

Hence:

$$
\|\vec{U}\|=\sqrt{\left(\sin \left(\frac{\beta}{2}\right) \cos \alpha \frac{d \beta}{d u}+2 \cos \left(\frac{\beta}{2}\right) \frac{d \alpha}{d u} \sin \alpha\right)^{2}+\left(2 \cos \left(\frac{\beta}{2}\right) \cos \alpha\right)^{2}\left\|\frac{d \hat{\beta}_{g}}{d u}\right\|^{2}}
$$

with 


$$
\left\|\frac{d \hat{\beta}_{g}}{d u}\right\|^{2}=\left(\frac{d \gamma}{d u}\right)^{2}
$$

so:

$$
\|\vec{U}\|=\sqrt{\left(\frac{d \beta}{d u} \sin \left(\frac{\beta}{2}\right) \cos \alpha+2 \frac{d \alpha}{d u} \cos \left(\frac{\beta}{2}\right) \sin \alpha\right)^{2}+\left(2 \frac{d \gamma}{d u} \cos \left(\frac{\beta}{2}\right) \cos \alpha\right)^{2}}
$$

Thus:

$$
\delta_{a}=\frac{0.886 \lambda_{0}}{T \sqrt{\left(\frac{d \beta}{d u} \sin \left(\frac{\beta}{2}\right) \cos \alpha+2 \frac{d \alpha}{d u} \cos \left(\frac{\beta}{2}\right) \sin \alpha\right)^{2}+\left(2 \frac{d \gamma}{d u} \cos \left(\frac{\beta}{2}\right) \cos \alpha\right)^{2}}}
$$

\subsection{Ellipse computation}

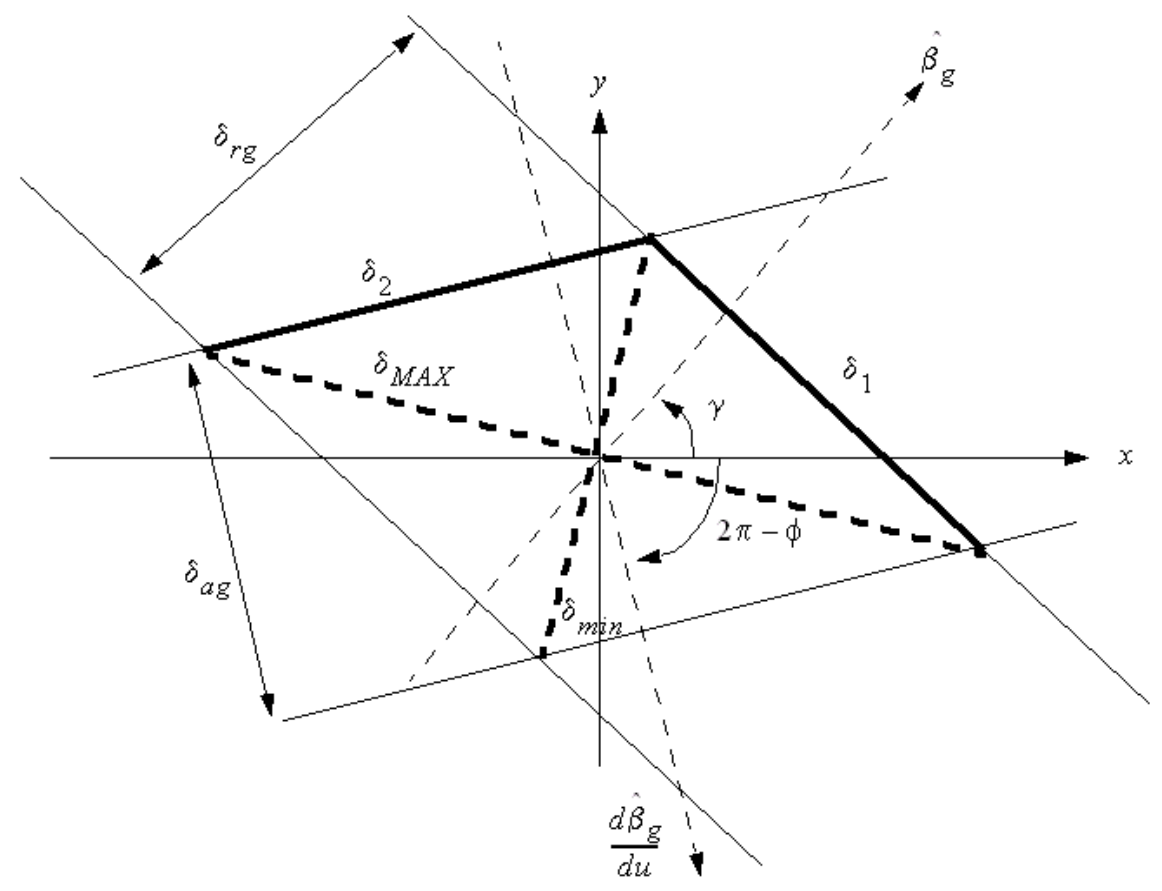

Figure 13: Ellipse resolution assembly

The first step is to compute the lengths $\delta_{1}$ and $\delta_{2}$ of the sides of the resolution parallelogram (figure 13). They are given by:

$$
\begin{aligned}
& \delta_{1}=\frac{\delta_{r}}{\sin \gamma} \\
& \delta_{2}=\frac{\delta_{a}}{\sin \gamma}
\end{aligned}
$$

Then, we evaluate the orientation $\gamma$ and $\phi$ of vectors $\vec{\beta}_{g}$ and $\frac{d \vec{\beta}_{g}}{d u}$ : 


$$
\gamma=\arctan \left(\frac{\overrightarrow{\beta_{g}} \cdot \hat{y}}{\vec{\beta}_{g} \cdot \hat{x}}\right)
$$

and for $\frac{d \vec{\beta}_{g}}{d u}$ :

$$
\phi=\arctan \left(\frac{\sin \alpha \sin \gamma \frac{d \alpha}{d u}-\cos \alpha \cos \gamma \frac{d \gamma}{d u}}{\sin \alpha \cos \gamma \frac{d \alpha}{d u}+\cos \alpha \sin \gamma \frac{d \gamma}{d u}}\right)
$$

The next step is to evaluate $\vec{\delta}_{\max }$ and $\vec{\delta}_{\min }$. To do this, we apply the following algorithm:

$$
\begin{aligned}
& \text { - if } \delta_{1}>\delta_{2} \text { then } \vec{\delta}_{\text {max }}=\left[\begin{array}{ll}
-\delta_{1} \sin \phi & \delta_{1} \cos \phi
\end{array}\right]^{T}, \vec{\delta}_{\text {min }}=\left[\begin{array}{ll}
\delta_{2} \cos \phi & \delta_{2} \sin \phi
\end{array}\right]^{T} \text {, else } \\
& \vec{\delta}_{\text {max }}=\left[\begin{array}{ll}
-\delta_{2} \sin \gamma & \delta_{2} \cos \gamma
\end{array}\right]^{T} \text { and } \vec{\delta}_{\text {min }}=\left[\begin{array}{ll}
\delta_{1} \cos \gamma & \delta_{2} \sin \gamma
\end{array}\right]^{T} .
\end{aligned}
$$

With these parameters, the ellipse equation is computed:

$$
\begin{aligned}
& x_{e}=\left\|\vec{\delta}_{\text {max }}\right\| \cos \Phi \\
& y_{e}=\left\|\vec{\delta}_{\text {min }}\right\| \sin \Phi
\end{aligned}
$$

with $0 \leq \Phi \leq 2 \pi$. Then the resolution ellipse in the image plane is evaluated:

$$
\begin{aligned}
& x_{\delta}=x_{e} \cos \left(\arg \left(\vec{\delta}_{\text {MAX }}\right)\right)-y_{e} \sin \left(\arg \left(\vec{\delta}_{\text {max }}\right)\right) \\
& y_{\delta}=x_{e} \sin \left(\arg \left(\vec{\delta}_{\text {MAX }}\right)\right)+y_{e} \cos \left(\arg \left(\vec{\delta}_{\text {max }}\right)\right)
\end{aligned}
$$

\section{References}

[1] N. Willis, Bistatic Radar. Artech Housse: New York, NY, USA, 1991.

[2] V. Giroux, H. Cantalloube, and F. Daout, "An omega-k algorithm for sar bistatic systems," in IGARSS'05. Proceedings of IGARSS, july 2005, pp. 1060 - 1063.

[3] — - "Frequency domain algorithm for bistatic sar," in EUSAR 2006, 2006.

[4] F. Comblet, F. Pellen, A. Baussard, and A. Khenchaf, "Bistatic sar: Theory and simulation," in IEEE Antennas and Propagation Society International Symposium, July 2005, pp. 664667.

[5] M. Cherniakov, R. Saini, R. Zuo, and M. Anoniou, "Space surface bistatic sar with spaceborne non-cooperative transmitters," in Proceedings of the 2005 Radar Conference EURAD, October 2005, pp. 9-12. 
[6] H. Cantalloube, M. Wendler, V. Giroux, P. Dubois-Fernandez, and R. Horn, "A first bistatic airborne sar interferometry experiment - preliminary results," in 2004 Sensor Array and Multichannel Signal Processing Workshop, July 2004, pp. 667-671.

[7] M. Soumekh, "Bistatic synthetic aperture radar imaging using wide-bandwidth continuouswave sources," in SPIE Conference on Radar Processing, Technology, and Applications, July 1998, pp. 99-109.

[8] I. Walterscheid, J. H. G. Ender, A. R. Brenner, and O. Loffeld, "Bistatic sar processing and experiments," IEEE Transactions on Goescience and Remote Sensing, vol. 44, no. 10, pp. 2710-2717, October 2006.

[9] G. Ginolhac, F. Schmitt, F. Daout, and P. Forster, "Multifrequency and multistatic inverse synthetic aperture radar, with application to fm passive radar," EURASIP Journal on Advances in Signal Processing, vol. 2010, 2010.

[10] Y. Wu and D. M. Jr., Multistatic Synthetic Aperture Imaging of Aircraft using Reflected Television Signals, ser. Algorithms for Synthetic Aperture Radar Imaging VIII. Proc. SPIE 4382, Ed. E. G. Zelnio, april 2001.

[11] P. Howland, D. Maksimiuk, and G. Reitsma, "Fm radio bistatic radar," IEE Proc.-Radar, Sonar Navig., vol. 152, no. 3, pp. 107-115, june 2005.

[12] P. Howland, "Target tracking using television-based bistatic radar," IEE Proc.-Radar, Sonar Navig., vol. 146, no. 3, pp. 166-174, june 1999.

[13] T. Tsao, M. Slamani, P. Varshney, D. Weiner, and H. Schawarzlander, "Ambiguity function for a bistatic radar," IEEE Transactions on Aerospace and Electronic Systems, vol. 33, no. 3, pp. 1041-1051, july 1997.

[14] I. Bradaric, G. Capraro, D. D. Weiner, and M. C. Wicks, "Multistatic radar systems signal processing," in IEEE Conference on Radar, April 2006.

[15] R. Fogle and B. Rigling, "Optimal geometry designs for unconstrained and topologically constrained multistatic sensors," in Asilomar Conference on Signals, Systems and Computers, November 2006.

[16] A. Farina, F. Gini, M. Greco, and P. Stinco, "Optimal selection of the tx-rx pair in a multistatic radar system," in COGIS 09, Paris, November 2009.

[17] I. Bradaric, G. Capraro, and M. Wicks, "Sensor placement for improved target resolution in distributed radar systems," in IEEE Radar Conference, Rome, May 2008. 
[18] T. Zeng, M. Cherniakov, and T. Long, "Generalized approach to resolution analysis in bsar," IEEE Transactions on Aerospace and Electronic Systems, vol. 41, no. 2, pp. 461-474, April 2005

[19] M. Soumekh, Synthetic Aperture Radar Signal Processing with MATLAB Algorithms. Wiley, John \& Sons, April 1999.

[20] M. Richards, Fundamentals of radar signal processing. Mcgraw-hill professional publishing, june 2005.

[21] T. Derham, S. Doughty, C. Baker, and K. Woodbridge, "Ammbiguity function for spatially coherent and incoherent multistatic radar," IEEE Transactions on Aerospace and Electronic Systems, vol. 46, no. 1, pp. 230-245, january 2010.

[22] http://www.trimble.com/planningsoftware_ts.asp, 2007.

[23] D. Brian, D. Rigling, and R. L. Moses, "Polar format algorithm for bistatic sar," IEEE Transactions on Aerospace and Electronic Systems, vol. 40, no. 4, pp. 1147-1159, October 2004

Franck DAOUT received the M. S. degree and the Ph.D. degree in Electronics from the University of Nantes (France) in 1996. From 1997 to 1999, he was a Research Engineer at the French Naval Academy, (Brest, France), GTS (Groupe de Traitement du Signal) Laboratory in the SONAR team. In 1999, he becomes an Assistant Professor at the University of Paris Ouest Nanterre la Defense. His researches and teaching courses are in the fields of electromagnetic wave propagation, microwave and signal processing. Currently, he works at the SATIE Laboratory (Systemes et Applications des Technologies de l'Information et de l'Enérgie, ENS Cachan, France) on RADAR system.

Françoise SCHMITT received the Ph.D. degree in Acoustics from the University Paul Sabatier of Toulouse (France) in 1993. From 1994 to 1997, she was a Research Engineer at the French Naval Academy, (Brest, France), laboratory GTS (Groupe de Traitement du Signal) in the SONAR team. In 1997, she becomes an Assistant Professor at the Ecole Centrale of Nantes to be on secondment at the French Naval Academy. From 2002 she becomes assistant professor at the University of Paris Ouest Nanterre la defense. Her teaching courses are in electronic and in physic and her researches are in the fields of acoustic and electromagnetic wave propagation. Currently, she works at the SATIE Laboratory (Systemes et Applications des Technologies de l'information et de l'énérgie, ENS Cachan, France) on RADAR system.

Guillaume GINOLHAC was born in France in 1974. He received the degree of Electrical Engineer from the Ecole Nationale Supérieure d'Ingénieurs Electriciens de Grenoble (ENSIEG) in 
1997, and the Ph.D. degree in Signal Processing from the Institut National Polytechnique de Grenoble (INPG) in 2001. He is currently Maitre de Conférences of electrical engineering at the Institut Universitaire de Technologie de Ville d'Avray, Université Paris X, France. He is also a researcher of the SATIE Laboratory from ENS Cachan. His research interests are in estimation and detection theory with applications to array processing and radar/sonar.

Philippe FARGETTE born July, 7 1965, in France, received the Dipl. Ing in engineering mechanics from ENSMM and the Master degree in acoustics from Besancon University, in 1989. In 1990, he was with the Structural Dynamics Department of ONERA, Palaiseau, France and worked on identification techniques and flutter calculation. He joined the Electromagnetic and Radar Department of ONERA in 2003. His studies cover passive radar and radar simulation framework. 